\title{
Epidemiological, Clinical, Diagnostical and Histopathological Aspects of Medular Compressions of Metastatic Origin
}

\author{
Jean Paul Engbang ${ }^{1,2, *}$, Mathieu Motah ${ }^{1,3}$, Basile Essola ${ }^{1}$, Landry Metso ${ }^{1}$ \\ ${ }^{1}$ Faculty of Medicine and Pharmaceutical Sciences, The University of Douala, Douala, Cameroun \\ ${ }^{2}$ Douala Laquintinie Hospital, Douala, Cameroun \\ ${ }^{3}$ Douala General Hospital, Douala, Cameroun
}

Email address:

jean_pen@yahoo.ca (J. P. Engbang), jpauleng@gmail.com (J. P. Engbang)

${ }^{*}$ Corresponding author

\section{To cite this article:}

Jean Paul Engbang, Mathieu Motah, Basile Essola, Landry Metso. Epidemiological, Clinical, Diagnostical and Histopathological Aspects of Medular Compressions of Metastatic Origin. Cancer Research Journal. Vol. 9, No. 1, 2021, pp. 34-40. doi: 10.11648/j.crj.20210901.15

Received: January 26, 2021; Accepted: February 3, 2021; Published: February 10, 2021

\begin{abstract}
Background: according to the World Health Organization (WHO) in 2019, cancer metastasis constitutes a fast proliferation of abnormal cells which spread to other organs. Bone is the third most targeted organ behind the lungs and the liver, and the most frequent bone's localizations are; the spine, pelvic bone and the ribs. Metastatic spinal compressions constitutes, a well-defined clinical entity of which the prevalence and incidence depends on the primitive tumor, Objective: To study the epidemiological, clinical, diagnosis and the histopathologic aspect of spinal cord metastatic compressions. Patients and method: It was a retrospective descriptive study over a period 10 years in the Douala General and Laquintinie Hospitals. We included in our study patients with slow spinal compression since January 1st 2010 to December 31st 2019. We included in our study, filled patients medical reports suffering of a primitive tumor which spread to the spine, with pathological and radiological evidence. Results: we recruited a total of 125 patients of which $68 \%$ where male and $32 \%$ female with a sex-ratio of 2.12, the mean age was $55.8 \pm 15.397$. The most frequent spine localization of metastasis was the lumbar region $57.1 \%$. The spine pain was the main symptom seen in $98.4 \%$ associated to radicular nerf pain in $33.6 \%$, sensibility and motor dysfunctions represented $94 \%$ and $92 \%$ respectively, and urinary dysfunction represented $44.8 \%$. The diagnosis was essentially based on spine X-rays and was being realized in $64.8 \%$ of cases. Lytic lesions and bone condensation were the main abnormalities viewed with $22.4 \%$. The second group of imaging was the CT-Scan and MRI done in $62.4 \%$. The most represented primitive tumor was the prostate cancer 59 cases $(47.25 \%)$ with adenocarcinoma as the main histological type 56 cases. Breast cancer came next with 32 cases (25.6\%), invasive ductal carcinoma as the most represented histological type 26 cases (20.6\%). The lung cancer came at the third position with 7 cases (10.4\%) with the small cell carcinoma been the most represented histological type with $4.8 \%$. Conclusion: spinal cord compression of metastatic origin is a frequent pathology in patients with osteophilic tumor, despite adequate measures, the prognosis remains unresolved. Emphasis should be placed on raising awareness of different early diagnostic measures.
\end{abstract}

Keywords: Spinal Metastasis, Spinal Cord Compression, Emergency

\section{Introduction}

According to the World Health Organization (WHO) 2019 , metastases are the detachment of cancer cells from a primary tumor that will swarm to another part of the body using lymphatic vessels or blood vessels [1]. Its locations are multiple and can occur on several organs. Bone is the third most affected organ after the lung and liver [2]. The most frequent bony locations are: the spine, pelvis and ribs [2]. Spinal cord compression is any injury starting from the spinal or intraspinal cord compressing the spinal cord and gradually leading to loss of function [3]. 
Metastatic lesions of the spine are a very disabling entity, on the one hand because of the pain they induce and on the other hand because of the neurological deficit [4]. Worldwide, the incidence of metastases varies depending on the location of the primary cancer [5]. A study conducted in the United States by Domchek et al showed a rate of metastasis in the spine of $19.6 \%$ of the various primary cancers distributed as follows: breast cancer $8.4 \%$ myeloma $3.6 \%$ cancer of the prostate $2.4 \%$ and other $5.2 \%$ (lung cancer, kidney cancer, cervical cancer, etc.) [6].]. The work done in Morocco by R. Karim et al showed that the average age of onset of spinal metastases was 46 years with a predominance of men; the primary tumor was bronchogenic cancer in men and breast carcinoma in women [7]. Clinically radicular pain is found at $94 \%$, lumbar instability at $10 \%$, numbness at $79 \%$, retention problems and urinary incontinence at $61 \%$ [8]. Paraclinical diagnosis is currently facilitated by standard radiography and CT scan of the spine which could objectify bone lysis. Medullary MRI shows the impact on the marrow [9]. The definitive diagnosis is obtained by surgical biopsy after laminectomy or a fine needle aspiration [10]. According to the French Neurosurgery Society, the main treatment for spinal metastases is surgery. Radiotherapy and postoperative chemotherapy are used respectively if the primary tumor is radioactive and chemosensitive [11]. Spinal cord compression of metastatic origin is an oncological and neurosurgical emergency and is a common complication of cancer. Survival after the onset of malignant spinal metastases is reduced with the onset of motor and sensory neurological deficits, spinal cord ischemia with edema [4]. The scarcity of studies in Africa in general and in Cameroon in particular aroused our interest in this study with the aim of contributing to the management of spinal cord compression of metastatic origin in Cameroon.

\section{Material and Methods}

It was a descriptive study with retrospective data collection, carried out in the departments of surgery, oncology, neurology, anatomo-pathology, radiology, radiotherapy of the General and Laquintinie hospitals in Douala, over a period of 10 years (January 2010 - December 2019). We went to the archives of the centers where the patient files were stored. We selected the files of patients with slow spinal cord compression, from which we selected the charts of patients with metastatic spinal cord compression with pathological and radiological evidence. The variables collected were: age, sex, history (diabetes, hypertension, cancer and other chronic pathologies), characteristics of pain, motor deficits, sensory deficits, sphincter disorders, results of laboratory tests and radiological, histological types of primary tumors. The data collected were recorded and analyzed by the computer statistical software Epi Info version 3.5.4.

\section{Results}

\subsection{Sociodemographic Data}

\subsubsection{Age}

As shown in figure 1, the age of the population varied between 12 and 84 years with an average of $55.8 \pm 15.397$ years. The most represented age group in our series was between $62-72$ years 36 cases $(28.8 \%)$.

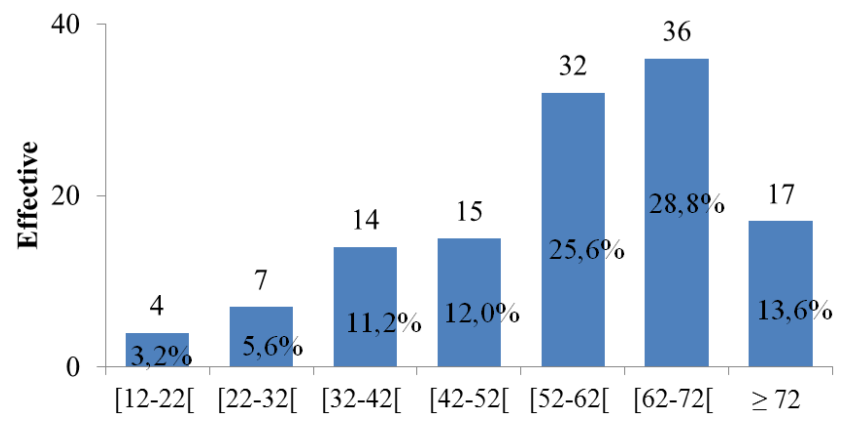

Figure 1. Distribution of patients according to age groups.

\subsubsection{Sex}

A total of $85(68.0 \%)$ were male and $40(32.0 \%)$ were female, for an $\mathrm{M} / \mathrm{F}$ sex ratio of 2.12 .

\subsection{Clinical Aspects}

\subsubsection{History (Known Tumor Pathology)}

The most represented cancer was that of the prostate 24 cases (19.2\%) with adenocarcinoma as histological type, followed by breast cancer 12 cases $(9.6 \%)$ with ductal carcinoma as histological type. (Table 1).

Table 1. History (known tumor pathology).

\begin{tabular}{lll}
\hline Tumor pathology & Effectif $(\mathbf{N}=\mathbf{1 2 5})$ & Pourcentage $(\%)$ \\
\hline Prostate cancer & 24 & 19.2 \\
Adenocarcinoma & 24 & 19.2 \\
Breast cancer & 12 & 9.6 \\
Ductal carcinoma & 12 & 9.6 \\
Lung cancer & 1 & 0.8 \\
Small cell carcinoma & 1 & 0.8 \\
Thyroid cancer & 1 & 0.8 \\
Papillary carcinoma & 1 & 0.8 \\
Non-Hogkin lymphoma & 1 & 0.8 \\
Cancer of the cervix & 1 & 0.8 \\
Squamous cell carcinoma & 1 & 0.8 \\
\hline
\end{tabular}

\subsubsection{Evolution Period}

In our study, 12 cases had an evolution period of less than one month (time between the discovery of cancer and the appearance of spinal metastasis), 23 cases (57.5\%) 1 to 6 months, 20 cases between 6 and 12 months and 10 patients an evolution period beyond 12 months.

\subsubsection{Spinal Disorders}

Spinal pain was found in 123 cases $(98.4 \%)$; lumbar pain was represented in 89 cases $(66.4 \%)$, chest pain in 38 cases (28.4\%) and neck pain in 7 cases (5.2\%) (table 2). 
Spinal stiffness was present in 23 cases $(18.4 \%)$ followed by damage to spinal statics found in 17 cases $(13.6 \%)$.

Table 2. Distribution according to spinal disorders.

\begin{tabular}{lll}
\hline Spinal disorders & Effective & Percentage ((\%) \\
\hline Spinal pain & & \\
Lumbar pain & 89 & 66.4 \\
Thoracic pain & 38 & 28.4 \\
Neck pain & 7 & 5.2 \\
Other spinal disorders & & \\
Spinal stiffness & 23 & 18.4 \\
Kyphosis & 7 & 5.6 \\
Scoliosis & 4 & 3.2 \\
Lordosis & 3 & 2.4 \\
Spinal static damage & 17 & 13.6 \\
\hline
\end{tabular}

\subsubsection{Lesion Syndrome}

Radicular pain was encountered in 42 cases $(33.6 \%)$ with sciatica in 30 cases $(24 \%)$, cervico-brachial neuralgia in 5 cases $(4 \%)$, cruralgia in 5 cases $(4 \%)$ and neuralgia intercostal in 2 cases $(1.6 \%)$ (table 3$)$

Table 3. Distribution of patients according to other types of disorders.

\begin{tabular}{lll}
\hline Disorders & Effective (N=125) & Percentage (\%) \\
\hline Root pain & 30 & 24.0 \\
Sciatica & 5 & 4.0 \\
Cervico-brachial neuralgia & 2 & 1.6 \\
Intercostal neuralgia & 5 & 4.0 \\
Cruralgia & & \\
Sub-lesion syndrome & 69 & 59.0 \\
Paresthesia & 38 & 32.0 \\
Hypoesthesia & 8 & 6.4 \\
Anesthesia & 2 & 1.6 \\
Hypesthesia & & \\
Motor disorders & 51 & 40,8 \\
Paraparesis & 48 & 38,4 \\
Paraplegia & 5 & 4.0 \\
Hemiplegia & - & - \\
Hemiparesis & 4 & 3.2 \\
Monoplegia & - & - \\
Monoparesis & 1 & 0.8 \\
Tetraparesis & 6 & 4.8 \\
Tetraplegia & & \\
Reflex disorders & 56 & 32.0 \\
Abolished osteotendinous reflex & 40 & 13.6 \\
Babinski sign & 17 & 3.2 \\
Abolished cutaneous-abdominal & 4 & 4.8 \\
reflex & 51 & \\
Sphincter disorders & 5 & \\
Sphincter disorders & & \\
Urinary incontinence & & \\
Retention disorders & & \\
\hline & & \\
& & \\
\hline
\end{tabular}

Sensory disorders were found in 117 cases $(93.6 \%)$, more marked by paresthesia in 69 cases $(59.0 \%)$, hypoesthesia in 38 cases $(32.0 \%)$, anesthesia in 8 cases $(6,4 \%)$ and hyperesthesia in 2 cases $(1.6 \%)$.

Motor disorders were found in 115 cases (92\%) marked by paraparesis in 51 cases $(40.8 \%)$, paraplegia in 48 cases $(38.4 \%)$ and quadriplegia in 6 cases $(4.8 \%)$.

In our study, abolished osteotendinous reflexes were found in 40 cases $(32 \%)$, Babinski's sign was found in 17 cases $(13.6 \%)$ and the cutaneous-abdominal reflex was abolished in 4 cases $(3.2 \%)$.
Sphincter disorders were found in 56 cases $(44.8 \%)$ more marked by urinary incontinence in 51 cases $(40.8 \%)$ followed by retention disorders in 5 cases $(4.0 \%)$ (Table 3 )

\subsection{Paraclinical Data}

\subsubsection{Data from Standard Radiography, CT Scan and MRI of the Spine}

As shown in table 4, radiography was performed in 81 cases $(64.8 \%)$; the images showed that lytic lesions were represented in 28 cases $(22.4 \%)$ located mainly on the lumbar vertebrae in 15 cases $(12 \%)$, followed by osteocondensation lesions 28 cases $(22.4 \%)$ predominant on the lumbar vertebrae 18 cases $(14.4 \%)$.

CT and MRI were performed in 115 cases (92\%) and the radiological image the most found was bone lysis in 44 cases (32\%) with localization in the lumbar spine 26 cases $(20.8 \%)$

Table 4. Lesions according to X-ray, CT scan and MRI.

\begin{tabular}{lllllll}
\hline \multirow{2}{*}{ Radiological lesions } & \multicolumn{3}{c}{ Cervical spine } & \multicolumn{2}{c}{ Dorsal spine } & \multicolumn{2}{c}{ Lumbar spine } \\
\cline { 2 - 7 } & $\mathbf{n}$ & $\mathbf{\%}$ & $\mathbf{n}$ & $\mathbf{\%}$ & $\mathbf{n}$ & $\%$ \\
\hline X-ray & & & & & & \\
Bone lysis & 1 & 0,8 & 12 & 9,6 & 15 & 12 \\
Osteocondensation & 2 & 1,6 & 8 & 6,4 & 18 & 14,4 \\
Vertebral compaction & 1 & 0,8 & 6 & 4,8 & 13 & 10,4 \\
Mixed & 1 & 0,8 & 1 & 0,8 & 3 & 2,4 \\
CT scan / MRI & & & & & & \\
Compressions & 2 & 1,6 & 15 & 12 & 22 & 17,6 \\
Bone lysis & 4 & 3,2 & 14 & 11,2 & 26 & 20,8 \\
Osteocondensation & 1 & 0,8 & 7 & 5,6 & 14 & 11,2 \\
Vertebral compaction & 1 & 0,8 & 17 & 13,6 & 19 & 15,2 \\
Epiduritis & 0 & 0 & 2 & 1,6 & 3 & 2,4 \\
Mixed & 1 & 0,8 & 3 & 2,4 & 4 & 3,2 \\
\hline
\end{tabular}

\subsubsection{Bone Scintigraphy}

In our series, bone scintigraphy was found in 18 files (14.4\%); the lesion found was localized hyperfixation in the lumbar spine in 13 cases $(10.4 \%)$ followed by the dorsal spine in 4 cases $(3.2 \%)$.

\subsubsection{Location of Spinal Metastases}

In our series, the vertebral location of spinal metastases the most found was lumbar 88 cases $(57.1 \%)$ followed by thoracic location 58 cases $(37.7 \%)$ and finally the cervical spine with 8 cases $(3.2 \%)$

\subsubsection{Chest x-ray, Abdominal Ultrasound and Thoraco-Abdomino-pelvic Scanner Data}

Table 5 shows that, the chest X-ray was normal in 7 cases (5.6\%) and abnormal in 21 cases $(16.8 \%)$; Opacities were found in 6 cases $(4.8 \%)$, pleural effusions in 6 cases $(4.8 \%)$, micronodules in 5 cases $(4.0 \%)$, balloon release $(0.8 \%)$, nonquantifiable enlargement $(0.8 \%)$, atelectasis band $(0.8 \%)$ and posterior arch lysis $(0.8 \%)$.

We found 7 cases $(5.6 \%)$ of homogeneous hepatomegaly, 2 cases $(2.4 \%)$ of secondary hepatic localizations, 2 cases (1.6\%) of splenomegaly, 2 cases (1.6\%) lymph node involvement and 104 cases $(83.2 \%)$ of normal ultrasound.

In total, 86 cases $(68.8 \%)$ of thoraco-abdomino-pelvic CT 
scans are normal against 39 cases (31.2\%) abnormal including lung lesions in 14 cases $(11.2 \%)$, bone metastases in 9 cases $(7.2 \%)$, pelvic metastases in 6 cases $(4.8 \%)$, liver metastases in 5 cases $(4.0 \%)$, lymph node metastases in 3 cases $(2.4 \%)$ and peritoneal metastases in 2 cases $(1.6 \%)$.

Table 5. Results from chest X-ray, abdominal ultrasound and thoracoabdomino-pelvic scanner.

\begin{tabular}{|c|c|c|}
\hline Variable & Effective ( $N=125)$ & Percentage (\%) \\
\hline \multicolumn{3}{|l|}{ Chest x-ray } \\
\hline Normal & 7 & 5.6 \\
\hline Abnormal & 21 & 16.8 \\
\hline Opacity & 6 & 4.8 \\
\hline Pleural effusion & 6 & 4.8 \\
\hline Micronodule & 5 & 4.0 \\
\hline Balloon release & 1 & 0.8 \\
\hline Non-quantifiable enlargement & 1 & 0.8 \\
\hline Electasia band & 1 & 0.8 \\
\hline Lysis of the posterior arch & 1 & 0.8 \\
\hline \multicolumn{3}{|l|}{ Abdominal ultrasound } \\
\hline Normal & 104 & 83.2 \\
\hline Abnormal & 14 & 11.2 \\
\hline Homogeneous hepatomegaly & 7 & 5.6 \\
\hline Secondary hepatic location & 2 & 1.6 \\
\hline Splenomegaly & 2 & 1.6 \\
\hline Lymph node involvement & 2 & 1.6 \\
\hline Liver metastasis & 1 & 0.8 \\
\hline \multicolumn{3}{|c|}{ Thoraco-abdomino-pelvic scanner } \\
\hline Normal & 86 & 68.8 \\
\hline Abnormal & 39 & 31.2 \\
\hline Secondary lung injury & 14 & 11.2 \\
\hline Bone metastasis & 9 & 7.2 \\
\hline Pelvic metastasis & 6 & 4.8 \\
\hline Liver metastasis & 5 & 4.0 \\
\hline Lymph node metastasis & 3 & 2.4 \\
\hline Peritoneal metastasis & 2 & 1.6 \\
\hline
\end{tabular}

\subsubsection{Other Locations of Metastases}

As shown in table 6, among the other metastatic locations, bone metastases (ribs, sternum, collarbones and iliac bone) were the most represented location with 28 cases $(22.4 \%)$, pulmonary locations 19 cases $(15.2 \%)$, hepatic locations 10 cases $(8.0 \%)$, cerebral locations 5 cases $(4.0 \%)$, lymph node locations 3 cases $(2.4 \%)$, peritoneal locations 2 cases $(1.6 \%)$, bladder locations 2 cases $(1.6 \%)$ and finally the locations of the spleen and pelvis represented with one case respectively for a percentage of 0.8 each

Table 6. Distribution according to others locations of metastasis.

\begin{tabular}{lll}
\hline Others locations of metastases & Effective $\mathbf{( N = 1 2 5 )}$ & Percentage (\%) \\
\hline Costal & 14 & 11.2 \\
Hipbone & 8 & 6.4 \\
Clavicle & 2 & 1.6 \\
Sternum & 4 & 3.2 \\
Lungs & 19 & 15.2 \\
Liver & 10 & 8.0 \\
Brain & 5 & 4.0 \\
Ganglia & 3 & 2.4 \\
Peritoneum & 2 & 1.6 \\
Bladder & 2 & 1.6 \\
Spleen & 1 & 0.8 \\
Pelvis & 1 & 0.8 \\
\hline
\end{tabular}

\subsubsection{Biological Data}

In our series 94 cases $(76.4 \%)$ had a normal white blood cell count against 29 abnormal cases $(23.6 \%)$; 81 cases (65.9\%) had normal hemoglobin levels compared to 42 cases (34.1\%). 60 cases (92.3\%) had an abnormal PSA level and 5 cases $(7.7 \%)$ had a normal level. 112 cases $(89.6 \%)$ had an abnormal calcium level compared to 13 normal cases (10.4\%). 8 cases $(57.1 \%)$ had normal SV against 6 cases (42.9\%) abnormal, 17 cases $(85.0 \%)$ had abnormal CRP against 3 cases $(15.0 \%)$ normal. 2 cases $(100 \%)$ had a normal CA19.9 level (table 7)

Table 7. Effective according to biological assessment data.

\begin{tabular}{llll}
\hline Biological data & & Effective & Percentage (\%) \\
\hline WBC (123) & Normal (4-10) & 94 & 76.4 \\
& Abnormal & 29 & 23.6 \\
Hb (123) & Normal (9-14) & 81 & 65.9 \\
& Abnormal & 42 & 34.1 \\
& & & \\
PSA (65) & Normal (<6) & 5 & 7.7 \\
& Abnormal & 60 & 92.3 \\
Calcemia (125) & Normal (95-100) & 13 & 10.4 \\
& Abnormal & 112 & 89.6 \\
SR (14) & & & \\
& Normal (70/24h) & 8 & 57.1 \\
& Abnormal & 6 & 42.9 \\
CRP (20) & Normal (<6) & 3 & 15.0 \\
& Abnormal & 17 & 85.0 \\
& & & 0.0 \\
\hline
\end{tabular}

SR: Sedimentation Rate

\subsubsection{Certainty Diagnosis}

We found 125 files $(100 \%)$ in which the patients had performed a post-laminectomy biopsy.

\subsubsection{Histological Types (Primary Cancers)}

The most common primary cancer was prostate cancer in 54 cases $(43.2 \%)$ with adenocarcinoma as the most common histological type in 52 cases $(41.6 \%)$, which had the lumbar spine as the site of preferential metastasis in 41 cases of metastases (26.65\%). Breast cancer was the second most common primary tumor in 29 cases (23.2\%); ductal carcinoma was its main histological type in 24 cases $(19.2 \%)$ and lumbar spine in 18 cases $(17.6 \%)$ its preferred site of metastasis (table 8).

\section{Discussion}

The mean age of the population was $55.8 \pm 15.397$ years with extremes of 12 and 84 years. These results are similar to those of Y. Matsuyama et al in Japan, Marc C. Chamberlain in California, and Djiencheu et al in Cameroon who found an average age of 52 years, 41.3 years, and 42.5 years 
respectively. with respective extremes of 26 years and 77 years, 11 and 70 years, 06 and 75 years [12-14]. This can be explained by the fact that cancers grow in an insidious way.

Table 8. Primary cancers and their histological types depending on the location of the spinal metastases.

\begin{tabular}{|c|c|c|c|c|c|c|}
\hline \multirow[t]{2}{*}{ Cancers and histological types } & \multicolumn{2}{|c|}{$\begin{array}{l}\text { Cervical } \\
\text { spine }\end{array}$} & \multicolumn{2}{|c|}{$\begin{array}{l}\text { Dorsal } \\
\text { spine }\end{array}$} & \multicolumn{2}{|c|}{$\begin{array}{l}\text { Lumbar } \\
\text { spine }\end{array}$} \\
\hline & $\mathrm{n}$ & $\%$ & $\mathrm{n}$ & $\%$ & $\mathrm{n}$ & $\%$ \\
\hline Lung cancer & 1 & 0.65 & 4 & 2.6 & 4 & 2.6 \\
\hline Small cell cancer & 1 & 0.65 & 3 & 1.95 & 3 & 1.95 \\
\hline Neuroendocrine carcinoma & - & - & 1 & 0.65 & 1 & 0.65 \\
\hline Prostate cancer & 3 & 1.95 & 17 & 11.05 & 41 & 26.65 \\
\hline Adenocarcinoma & 3 & 1.95 & 16 & 10.4 & 38 & 24.7 \\
\hline Neuroendocrine carcinoma & - & _ & 1 & 0.65 & 1 & 0.65 \\
\hline Kidney cancer & - & - & 1 & 0.65 & 3 & 1.95 \\
\hline Clear cell carcinoma & - & - & 1 & 0.65 & 2 & 1.3 \\
\hline Nephroblastoma & - & - & - & - & 1 & 0.65 \\
\hline Breast cancer & 1 & 0.65 & 13 & 8.45 & 18 & 11.7 \\
\hline Ductal carcinoma & 1 & 0.65 & 11 & 7.15 & 14 & 9.1 \\
\hline Lobular carcinoma & - & - & 2 & 1.3 & 3 & 1.95 \\
\hline Thyroid cancer & 1 & 0.65 & 2 & 1.3 & 2 & 1.3 \\
\hline Papillary carcinoma & 1 & 0.65 & 1 & 0.65 & 1 & 0.65 \\
\hline Vesicular carcinoma & - & - & 1 & 0.65 & 1 & 0.65 \\
\hline Cervical cancer & - & - & 4 & 2.6 & 3 & 1.95 \\
\hline Squamous cell carcinoma & - & - & 1 & 0.65 & 3 & 1.95 \\
\hline Non-Hodgkin lymphoma & 1 & 0.65 & 3 & 1.95 & 4 & 2.6 \\
\hline Nasopharyngeal cancer & - & - & 3 & 1.95 & 2 & 1.3 \\
\hline Digestive cancer & - & - & 3 & 1.95 & 3 & 1.95 \\
\hline NSC of the groin & - & - & 1 & 0.65 & 1 & 0.65 \\
\hline $\begin{array}{l}\text { Left anterior mediastinal } \\
\text { neuroendocrine tumor }\end{array}$ & - & - & - & - & 1 & 0.65 \\
\hline No primitive tumor & 1 & 0.65 & 6 & 3.9 & 5 & 3.25 \\
\hline
\end{tabular}

NSC-Non-Small carcinoma.

Our sample had more men $68.0 \%$ than women $32.0 \%$ for a sex ratio $\mathrm{M} / \mathrm{F}$ of 2.12. These results are similar to those given by Y. Matsuyama et al in Japan in 2009, Kone et al in Mali and Djiencheu et al in Cameroon who had respectively found more men $56 \%, 68.75 \%, 64 \%$ with the sex ratio respective $\mathrm{M} / \mathrm{F}$ of $1.26 ; 2.2 ; 1.80[12,14,15]$. This may be partly explained by the prevalence of prostate and lung cancer in men.

Spinal pain reflects the suffering of the osteo-discoligamentous elements of the spinal canal, segmental stiffness of the spine easier to demonstrate in the cervical and lumbar areas which are normally more mobile than the dorsal region and pain caused by the pressure of the processes in spinous and paravertebral muscles. These signs constitute the spinal syndrome which is more frequent in lesions of the spine but which may accompany a juxta or even intramedullary tumor. In our series spinal pain was presented in 123 cases $(98.4 \%)$ with lumbar pain in 89 cases $(66.4 \%)$ chest pain in 38 cases (28.4\%) and neck pain in 7 cases (5.2\%). These results are similar to those of Zeleli et al in the United States in 2007, Kone et al in Mali, and Ismael et al in Morocco who found spinal pain in $60 \%, 75 \%, 85.6 \%$, respectively [15-17]. This can be explained by the fact that back pain is one of the telltale signs of spinal cord compressions.

Radicular pain is fixed, tenacious and unilateral at the beginning of metameric radicular topography, of variable intensity, often nocturnal or with nocturnal recrudescence. The radicular syndrome will be clearer in the cervical region where the radicular territory is well individualized and better explored, whereas in the thoracic region it can be reduced clinically to intercostal pain in the hemi-girdle [18]. Radicular pain was represented in 42 cases (33.6\%) with sciatica in 30 cases $(73 \%)$ cervico-brachial neuralgia in 5 cases $(12 \%)$ cruralgia in 5 cases $(12 \%)$ and intercostal neuralgia in 2 cases $(1.6 \%)$. These results are similar to those of Younes et al in Morocco in 2016 and Alkadi et al in Algeria in 2017 who found respectively that radicular pain was represented in $32 \%$ and $30 \%$ broken down respectively as follows: sciatica in $66.7 \%$ and $66.6 \%$ cervicobrachial neuralgia in $18.3 \%$ and 16.66 cruralgia in $8.3 \%$ and $16.66 \%$ intercostal neuralgia in $6.7 \%$ and $0 \%[17,19]$. This pain is often poorly described and reflects the suffering of the directly compressed metamer and is therefore capital because it readily allows locate the level to be explored radiologically.

Often delayed in relation to motor signs, are of great importance for the diagnosis of spinal cord compressions. In our series, sensory disorders were represented in 117 cases (93.6\%), more marked by paresthesia in 61 cases $(53.0 \%)$, numbness in 38 cases (33.0\%) and anesthesia in 18 cases (15.7\%). These results are not similar to those of Djiencheu in Yaounde (Cameroon) in 2007 and Kone et al in Mali, who found sensory disorders respectively in $59.7 \%$ and $56.6 \%$ of cases $[14,15]$; this can be explained by the fact that the patients recruited had already undergone slow spinal compressions. Moreover, Diomande et al in Côte d'Ivoire in 2015 on slow spinal cord compressions found similar results with sensory disorders reported in $78.5 \%$ of cases [20]. These disorders are essentially subjective; data from the literature show that these signs are reported in 20.5 to $50 \%$.

Motor disorders were represented in $92 \%$ of cases marked by paraparesis 61 cases $(48.8 \%)$, paraplegia 48 cases $(38.4 \%)$ and quadriplegia 6 cases $(4.8 \%)$. Several studies had found similar results, in particular Djiencheu et al in Cameroon in 2007 who found motor disorders in $89.6 \%$, marked by paraparesis in $60 \%$ of cases, paraplegia in $31.7 \%$ of cases, tetraparesis in $6.7 \%$ of cases and quadriplegia in $1.7 \%$ of cases. Diomandé et al at the CHU de Cocody in Abidjan in 2015 on slow spinal cord compressions found motor disorders at 98.3\% [14, 20]. Younes et al in Morocco in 2016 on the management of spinal cord compressions of tumor origin found that motor disorders were present at 92\% [17]. They determine the urgency of the treatment because they mark the degree of compression of the spinal cord and they represent one of the first causes of consultation in neurosurgery in the event of spinal cord compression.

Sphincter disorders were represented in 56 cases $(44.8 \%)$, more marked by urinary incontinence in 51 cases $(40.8 \%)$ and retention disorders in 5 cases $(4.0 \%)$. Studies carried out by Djiencheu et al in Cameroon in 2007 and Diomande in Côte d'Ivoire in 2015 found similar results with respective sphincter disorders of $53 \%$ and $40.5 \%$ [14, 20]. In 2013, a study conducted by Kassegne et al in Togo on slow spinal cord compressions found sphincter disorders in 67\% [21]; 
this difference can be explained by the fact that the patients presented to the hospital in advanced stages of their pathologies. Relatively late sphincter disorders are limited for a long time to delayed urination, urgent and frequent urges to urinate and later retention with incontinence [22].

While plain front and lateral radiographs of the spine are still performed as a clearing up examination for spinal pain syndrome, this examination very often remains negative at the onset of a bone tumor, benign or malignant. It is in fact known that a reduction of at least $50 \%$ of the calcium load within a bone tissue is necessary for there to be a translation on conventional radiography. In our series, radiology was performed in $64.8 \%$ of cases, the images showed that lytic lesions were represented in 28 cases $(22.4 \%)$, osteocondensation lesions in 28 cases $(22.4 \%)$, vertebral compression in 20 cases $(16.0 \%)$ and mixed lesions in 5 cases $(4.0 \%)$. These results are similar to those of Younes et al in Morocco in 2016 on the management of tumor spinal cord compressions in which the radiology was performed at $87 \%$ with osteolytic images at $22 \%$, vertebral compression at $15.8 \%$ and osteocondensation at $6 \%$ [17]. A study conducted by Alkadi et al in Algeria in 2017 on vertebral metastases found discordant results with a completion rate of $40 \%$; the most prominent pathological aspect was 50\% vertebral compression followed by $40 \%$ bone lysis [19]. This can be explained by the fact that the $\mathrm{x}$-ray, not being a standard examination for the diagnosis of spinal compressions, was less performed.

CT and MRI of the spine were performed in 115 cases (92\%), with bone lysis in 44 cases $(32 \%)$, compression in 39 cases $(31.2 \%)$ and vertebral compression in radiological images. 29 cases $(23.2 \%)$; the site of the lesion was found in $100 \%$ of cases. These results are in disagreement with those of Djiencheu et al in 2007 in Yaounde (Cameroon) on slow spinal cord compressions of tumor and pseudotumoral origin in which the diagnostic exploration of choice was the myeloscanner $47.8 \%$ [14]. This could be explained by the unavailability of MRI. These results are similar to those of Younes et al in Morocco who found the site of compression in $100 \%$ of patients with osteolysis followed by an intracanal process as the main radiological image [17]. This can be explained by the fact that these examinations are standard for the diagnosis of spinal cord compression and always confirm the lesion topography

In our series, the most represented vertebral metastasis location was lumbar with 88 cases $(57.1 \%)$, followed by thoracic location in 58 cases $(37.7 \%)$ and cervical location in 8 cases $(5.2 \%)$. On the other hand, studies carried out in Cameroon (Yaounde) in 2007 by Djiencheu et al and in Morocco in 2016 by Younes et al found a rate of dorsal metastases at $55 \%$ and $52 \%$, lumbar $30 \%$, cervical $15 \%$ [14, 17]; this can be explained by the fact that their samples were smaller than ours. These results are similar with a study conducted by Alkadi et al in Algeria in 2017 on vertebral metastases, finding a rate of lumbar metastases of $50 \%$ and a rate of thoracic metastases of $40 \%$ [19]. This can be explained by the fact that the spine is a highly vascularized region rich in growth factor.

The chest x-ray has an important place in tumor pathology because it is necessary as part of an extension workup which is often for diagnostic purposes since the lung is the first target organ for metastasis. This examination is also performed in the pre-radiotherapy assessment. The chest $\mathrm{x}$ ray was normal in 7 cases $(5.6 \%)$ and abnormal in 21 cases $(16.8 \%)$ and opacities were found in 6 cases $(4.8 \%)$, pleural effusions in 6 cases $(4,8 \%)$, micronodules in 5 cases $(4.0 \%)$, balloon release $(0.8 \%)$, non-quantifiable enlargement $(0.8 \%)$, atelectasis band $(0.8 \%)$, posterior arch lysis $(0.8 \%)$. These results are similar to those of Younes et al in Morocco in 2016 and Alkadi et al in Algeria in 2017 who found respectively similar results with abnormal radiological image in 29 cases $(15.4 \%)$ and 18 cases $(20 \%)[17,19]$. This can be explained by the fact that the chest $\mathrm{x}$-ray is often replaced by the thoraco-abdomino-pelvic scanner.

Abdominal ultrasound has a key place in cancer disease with several advantages because it is inexpensive and accessible; it makes it possible to make the diagnosis of digestive metastases in occurrence hepatic metastases and peritoneal carcinomas. This examination is also used in the pre-radiotherapy assessment. In our series we had a completion rate of $94.4 \%$ with $11.2 \%$ of images which were pathological, namely 7 cases $(5.6 \%)$ of homogeneous hepatomegalies, 3 cases $(2.4 \%)$ of secondary hepatic locations, 2 cases $(1.6 \%)$ of splenomegaly, 2 cases $(1.6 \%)$ of lymph node involvement and 104 cases $(83.2 \%)$ of normal ultrasound. These results are similar to those of Younes et al in Morocco in 2016 on the management of spinal cord compressions of tumor origin who found $18 \%$ pathological image with secondary hepatic localizations and lymph node involvement [17]. On the other hand, these results are not similar to those of Alkadi et al in Algeria in 2017 on vertebral metastases which found that pathological images represented $30 \%$ of their number consisting only of liver metastases; this can be explained by the fact that their sample was lower than ours [19].

The thoraco-abdomino-pelvic scanner makes it possible to optimize the thoraco-abdomino-pelvic exploration in the search for secondary and lymph node lesions. The thoracoabdominal-pelvic scanner is essential in the study of cancerous disease. In our series 86 cases $(68.8 \%)$ of thoracoabdomino-pelvic CT scans are normal against 39 cases (31.2\%) abnormal among which pulmonary lesions 14 cases (11.2\%), bone metastases 9 cases $(7.2 \%)$, pelvic metastases 6 cases $(4.8 \%)$, liver metastases 5 cases $(4.0 \%)$, lymph node metastases 3 cases $(2.4 \%)$, peritoneal metastases 2 cases ( 1 , $6 \%$ ). These results are similar to those of Alkadi et al in Algeria in 2017 on vertebral metastases in which we had a $100 \%$ achievement rate with $30 \%$ pathological images (pulmonary metastases, hepatic metastases, tumor processes of the prostate, supra and sub diaphragmatic lymphadenopathy with multiple bone lesions) [19]. This can be explained by the fact that this examination is essential for an extension assessment.

The most common primary cancer was prostate cancer in 
54 cases $(43.2 \%)$, with adenocarcinoma as the most common histological type in 52 cases $(41.6 \%)$, followed by breast cancer in 29 cases $(23,2 \%)$ with ductal carcinoma as the most frequent histological type in 24 cases $(19.2 \%)$. These results are similar to several studies including Sidarnet et al in the United States, Younes et al in Morocco, Djiencheu et al in Cameroon which had found that prostate cancer and metastasized the highest level of the spine [14, 17, 23]. This can be explained by the fact that breast cancer is the most common cancer in women and prostate cancer the most common in men.

\section{Conclusion}

Spinal cord compressions of metastatic origin are frequent affections in oncology developing in elderly subjects with a predominance of men. The most common clinical signs are spinal pain, radicular pain, sensory disturbances, motor disturbances and sphincter disturbances. The most common metastatic location was lumbar followed by thoracic location. MRI and CT scans of the spine are the standard examinations because they allow the lesion topography to be determined. The most frequently found primary cancers are prostate cancer with adenocarcinoma as the main histological type, followed by breast cancer with ductal carcinoma as the main histological type.

\section{References}

[1] Ferlay J, Soerjomataram, Ervik M, Dikshit R, Eser R MC et al. GLOBOCAN 2012 v1.0, Cancer Incidence and Mortality worldwide: IARC CancerBase No. 11 Lyon, France: International Agency for Research on Cancer. Int J Cancer. 2015; 136 (5): E359-86.

[2] Samlali A, Bouchbika Z, Bennani W, Taleb A et al Brain metastasis from rectal adenocarcinoma: about a case and review of the literature. $2017 ; 26: 58$

[3] Camins MB, Jenkins AL, Singhal A PR. Tumor of vertebral axis: Begnin, primary malignant, and metastatic tumor. 5th ed Philadelphia WB Saunders Co. 2004; 4835-68.

[4] Dennis D, Dominguez E, Lauper N, Velastegui A, Reynolds J. Métastases de la colonne vertébrale : indications au traitement chirurgical. Rev Med suisse. 2016; 12: 2168-71.

[5] M. Gremaud D, Delouche C Mo. Traitement des métastases osseuses par les biphosphonates. Rev Med suisse. 2006; 2: 31127.

[6] Domchek SM, Younger j, Finkelstein DM SM. Predictor of skeletal complications in patients with metastatic breast carcinoma. Cancer. 2000; 89: 363-8.
[7] R. karim, T. El Hamdani, A. Maati, T. Mesbahi AC et al. Prise en charge des métastases rachidiennes. Masson SAS. 2013; 59: 263 .

[8] Agarwall J, Baum P. critères pour la palliation des métastases osseuses. IAEA. 2008; 12-4.

[9] Hamaoka T, Madewell, Podoloff DA. Bone imaging in breast cancer. JClinOncol. 2004; 22: 2942-53.

[10] Dur H, Janson V. Differenzierte Therapie metastatischer läsionen des knochens. arthritis und Rheuma. 2007; 3: 163-8.

[11] Lipton A. New approaches to trating and preventing bone metastases. Curr Opin Support Palliat Care. 2010; 26 (4): $178-81$.

[12] Matsuyama Y. al E. surgical results of intramedullary spinal cord tumor with spinal cord monitorring to guide extent of resection. J. neurosurg spine. 2009; 10 (5): 404-13.

[13] Chamberlain M. Kormanik. epidural spinal cord compression a single institution's retrospective experience. Neuro Oncol. $1990 ; 120-3$

[14] Djiencheu, Njamnshi, Ngandeu, al E. compressions médullaires lentes d'origines tumorale et pseudo-tumorale à yaounde. AFJ neurol sci. 2007; 26: 15-21.

[15] Kone I. prise en charge des tumeurs vertébro-médullaires dans le service de neurochirurgie du CHU de Gabriel Toure. bibliosante. 2011; 21.

[16] Zileli M, Coll. Primary tumors of the cervical spine: a retrospective review of 35 surgically managed cases. spine J. 2007; 7 (2): 165-73

[17] Ismail Y. prise en charge des compressions médullaires d'origine tumorale. Fac médécine Marrakech. 2016; 35: 44

[18] Ongolo-Zogo, Djientcheu, Njamnshi, Lekoubou, Eloundou, Gonsu. contribution de l'imagerie médicale dans le diagnostic étiologique des compressions lentes au Cameroun. J Afr Imag Med. 2006; 1: 25-34

[19] Wahab A abd el, Soufyane A, Djalel B. Métastases vertébrales. Fac Med TLEMCEN. 2017; 39

[20] Diomande M, Nseng-Nseng R ingrid, Kouame-Assouan A eric, All E. compresions médullaires lentes: prévalence et étiologie. Rev marocaine Rhumatol. 2015; 33: 27-30

[21] Kassegne, Sakiye, Kanossoua, Al E. prise en charge des compressions médullaires lentes au Togo. Med sante Trop. 2013; 23: 206-10

[22] Joe C. Ramendi P. Metastic epidural spinal cord compression. Lancet Neurol. 2008; 7 (5): 459-66.

[23] Sinardet D, Chabane A, Khalil T, Seigneuret E. Resultats neurologiques de 152 patients chirurgicaux atteints de métastases vertébrales. PubMed. 2000; 46 (1): 4-10. 